\title{
Fat eggs shape offspring health
}

Erica D. Watson and Joanna Rakoczy

Dept of Physiology, Development and Neuroscience, University of Cambridge, Cambridge, UK e-mail: edw23@cam.ac.uk

\begin{abstract}
How maternal diet influences offspring metabolism is unclear since it is difficult to distinguish between the effects of the in utero environment and epigenetic factors contributed by the oocyte. In a mouse model of high fat diet, a new study teases apart these mechanisms using IVF technology, and shows that susceptibility of offspring to metabolic disorder is likely attributed to epigenetic inheritance via the oocyte.
\end{abstract}

\section{Main Body}

Mounting evidence suggests that the metabolic status of parents leads to a greater susceptibility of metabolic disease in their offspring. While the worldwide prevalence of obesity and type 2 diabetes is ever increasing, there is a growing need to understand how parental diet alters the health of the next generation. Studies on the paternal effects of diet feature more prominently in the literature, the most recent of which demonstrate that malnourishment of male mice affects the expression of noncoding RNA in sperm ${ }^{1-3}$. This alone is sufficient to disrupt gene expression and metabolism in their offspring. Sperm DNA methylation patterns are also affected by $\operatorname{diet}^{4}$, though matching differentially methylated regions in sperm with metabolic phenotypes in the offspring can be challenging. Considerably more complications arise when studying the effects of maternal diet on the susceptibility of offspring to metabolic disruption and therefore, less is known. This is because it is difficult to separate the effects of physiology during pregnancy from altered epigenetic factors in the oocyte. In this issue, Johannes Beckers and colleagues ${ }^{5}$ address this problem by utilizing a mouse model of HFD together with in vitro fertilization (IVF) to directly show that maternal diet triggers a defect(s) in the oocyte, which programs metabolic disease in her offspring.

\section{Disease inheritance via gametes}

Huypens et al. ${ }^{5}$ set out to understand the gametic effects of parental HFD on offspring metabolic disease. To do this, they generated female and male mice that were obese, hyperinsulinemic during fasting, and severely glucose intolerant by feeding them a HFD for 6 weeks. To determine whether these metabolic traits were inherited by their offspring through germline effects, gametes of the parental generation were isolated for IVF after dietary exposure. Maternal and paternal effects were assessed by specific gametic combinations: oocytes from HFD-fed females were fertilized with 
sperm from males fed a normal diet, and sperm from HFD-fed males fertilized oocytes from females fed a normal diet. The authors then transferred the in vitro-derived embryos at the two-cell stage into the oviducts of surrogate females fed nothing but a normal diet. The IVF technique is beneficial to understanding this question because it eliminates the effects of diet on the maternal uterine environment since the unfertilized eggs were removed from the oviduct shortly after ovulation. IVF also excludes the seminal fluid as a factor since the sperm were collected from the epididymis prior to ejaculation. Providing the offspring with a surrogate mother also eliminated possible lactation and maternal behavioral effects caused by a maternal HFD. Therefore, any metabolic phenotypes observed in the resulting offspring would solely be attributed to defects in the gametes.

To determine the extent of metabolic disorder in the IVF-derived mice, the authors challenged the adult offspring with a HFD. Strikingly, sex-specific results were apparent. Female offspring derived from either a HFD-exposed oocyte or sperm were more likely to become obese compared to female offspring derived from gametes of mice fed a normal diet. Remarkably, female obesity was even more prevalent when both the sperm and egg came from HFD-fed parents indicating an additive effect. This differed in HFD-challenged male offspring, who became obese only when they were derived from a HFD-exposed oocyte. Both female and male offspring were glucose intolerant and insulin resistant but, again, only when their mothers were fed a HFD.

Overall, this study suggests that offspring metabolism is more sensitive to maternal obesity and diabetes than paternal, and that the offspring metabolic effect is attributed to factors within the oocyte independent from the mother's uterine environment.

\section{What's the mechanism?}

Although Huypens et al. ${ }^{5}$ do not identify the factor(s) altered in gametes by parental HFD, the results generated using IVF imply that the mechanism in oocytes is indeed epigenetic in nature. Further studies are required to explore the specific mechanism of inheritance. In support of an oocyte epigenetic factor influenced by diet, another independent model of nutrient deficiency shows inheritance of phenotypes via the maternal germline ${ }^{6}$. We commend the authors for analyzing large numbers of IVF derived-mice since multigenerational effects often lead to phenotypic variability in the offspring ${ }^{6}$ and require big populations to resolve. To generate such large cohorts, oocyte numbers were increased by superovulation, a process criticized by some for altering oocyte quality and inadvertently causing a maternal effect. However, the control offspring generated by the same method were not obese, glucose intolerant or insulin resistant ${ }^{5}$, thus adequately ruling out this possibility. 
It is known that placental insufficiency programs metabolic disease in the offspring ${ }^{7}$. In fact, maternal obesity in mice is associated with poor placentation ${ }^{8}$ as well as abnormal DNA methylation in oocytes ${ }^{9}$. Since placental development requires proper oocyte DNA methylation ${ }^{10}$, we speculate that maternal obesity disrupts the epigenetic landscape in oocytes leading to placental dysfunction and offspring metabolic disease. An oocyte not only contains genetic information but also provides the cellular material required for early development including RNAs and mitochondria, which are maternally derived and can have lasting effects on the offspring. Similar to in sperm ${ }^{1-3}$, the expression of non-coding RNAs may be altered in the oocyte by maternal diet to cause gene misexpression and metabolic changes in the next generation. Alternatively, mitochondrial inheritance may explain the effect of maternal obesity on offspring health since exposure of oocytes to an obese environment decreases mitochondrial DNA content ${ }^{11}$ and function $^{12}$. These defects persist in the mitochondria of fetal livers and kidneys ${ }^{11}$, thus potentially linking maternal diet to offspring metabolism via mitochondrial transmission. Pronuclear transfer experiments will help to distinguish mitochondrial effects from nuclear effects.

Genetic mutations alone cannot explain the drastic increase in prevalence of diabetes and obesity in the human population. Huypens et $\mathrm{al}^{5}$ and others ${ }^{1-4}$ demonstrate that epigenetic factors transmitted by both oocytes and sperm are responsive to parental diet and may be sufficient to cause metabolic disease in the next generation. The next step will be to undergo techniques used to identify epigenetic factors in sperm (e.g., methylome and transcriptome analyses) in combination with IVF to discover whether similar or novel epigenetic factors are present in oocytes.

\section{References}

1. Chen, Q. et al. Science 351, 397-400 (2016).

2. Grandjean, V. et al. Sci Rep 5, 18193 (2015).

3. Sharma, U. et al. Science 351, 391-6 (2016).

4. de Castro Barbosa, T. et al. Mol Metab 5, 184-97 (2016).

5. Huypens, P. et al. Nat Genet (2016).

6. Padmanabhan, N. et al. Cell 155, 81-93 (2013).

7. Barker, D.J. \& Thornburg, K.L. Clin Obstet Gynecol 56, 511-9 (2013).

8. Sasson, I.E. et al. Diabetologia 58, 615-24 (2015).

9. Hou, Y.J. et al. Sci Rep 6, 18858 (2016).

10. Branco, M.R. et al. Dev Cell 36, 152-63 (2016).

11. Wu, L.L. et al. Development 142, 681-91 (2015).

12. Igosheva, N. et al. PLoS One 5, e10074 (2010).

\section{Figure legend}

Figure 1. Huypens et al. ${ }^{5}$ determined that oocytes are responsive to maternal obesity and diabetes, and epigenetically transmit information to the next generation to cause metabolic disorder.

Although not identified, the factors may include DNA and/or histone methylation, non-coding RNA (e.g., microRNA or tRNA fragments), and mitochondria. 